\title{
Charge Transport in Hybrid Nanorod-Polymer Composite
}

\section{Photovoltaic Cells}

\author{
Wendy U. Huynh ${ }^{\ddagger}$, Janke J. Dittmer ${ }^{\ddagger}$, Nerayo Teclemariam, Delia \\ Milliron, A. Paul Alivisatos* \\ Department of Chemistry, University of California and Material Science Division, Lawrence \\ Berkeley National Laboratory, Berkeley CA 94720, USA \\ Keith W. J. Barnham \\ Department of Physics, Imperial College of Science and Technology, London SW7 2BW, \\ United Kingdom
}

Manuscript submitted to the Physical Reviews B on June 252002

PACS Codes: 73.50.-h, 73.50.Pz, 73.61.Ph, 73.63.Bd

*alivis@uclink4.berkeley.edu

These authors contributed equally to this work 


\begin{abstract}
Charge transport in composites of inorganic nanorods and a conjugated polymer is investigated using a photovoltaic device structure. We show that the current-voltage (I-V) curves in the dark can be modelled using the Shockley equation modified to include series and shunt resistance at low current levels, and using an improved model that incorporates both the Shockley equation and the presence of a space charge limited region at high currents. Under illumination the efficiency of photocurrent generation is found to be dependent on applied bias. Furthermore, the photocurrent-light intensity dependence was found to be sublinear. An analysis of the shunt resistance as a function of light intensity suggests that the photocurrent as well as the fill factor is diminished as a result of increased photoconductivity of the active layer at high light intensity. By studying the intensity dependence of the open circuit voltage for nanocrystals with different diameters and thus band gaps, it was inferred that Fermi-level pinning occurs at the interface between the aluminum electrode and the nanocrystal.
\end{abstract}




\section{INTRODUCTION}

Semiconductor nanocrystals are attractive as components of electronic devices because many of their physical properties can be controlled through modification of the diameter and shapes of these particles. Optoelectronic devices such as photovoltaic cells and light-emittingdiodes take advantage of the dependence of the band gap of nanocrystals on particle radius $\mathbf{b}$ absorb and emit light, respectively, with tunable wavelengths. Furthermore, a concentration of the density-of-states into quantized energy levels results in high oscillator strengths for high absorptivity or emissivity as compared with bulk values. Extensive development of the synthesis of colloidal nanocrystals, especially of CdSe, permits particles to be prepared with high yield and quality at relatively low temperatures $\left(\sim 300^{\circ} \mathrm{C}\right)$ in a wide range of diameters, aspect ratios and shapes ${ }^{1,2}$. Control of the surface passivation of the nanocrystals with organic ligands allow for the particles to be dissolved in a variety of solvents and dispersed in numerous polymers. The ease of processing nanocrystals is an advantage in the low cost fabrication of devices via spin casting, inkjet and screen printing, and other coating techniques from solution. Conjugated polymers share the same processing advantages with nanocrystals while further allowing for mechanical flexibility of the device film. Polymers have been used previously in photovoltaic cells, but the low electron mobilities of most conjugated polymers only allows them for use as the hole transporting component in a blend with other materials, such as fullerenes ${ }^{3}$, organic dyes $^{4,5}$ or in the present case with ranocrystals. In these composite materials, the photocurrent is typically increased by several orders of magnitude with regard to polymer only devices. By going from colloidal quantum dots to quantum rods the electron transport and hence the photocurrent can be further increased. Quantum rods allow for efficient one-dimensional electrical transport, which, as seen in $\mathrm{Si}$ nanowires, can reach carrier mobilities comparable to 
bulk values ${ }^{6}$. By combining nanorods with the conjugated polymer poly(3-hexylthiophene) (P3HT), we have obtained high efficiency solar cells with an Air Mass 1.5 Global (A.M. 1.5G) power conversion efficiency of $1.7 \%^{7}$.

In order to develop and improve device architectures that can be incorporated into useful electronic devices, it is necessary to understand i) the fundamental physical properties of the nanocrystal building blocks in composites with conjugated polymers and ii) the operating principles of such alternative devices. At the nanoscale many quantum mechanical processes become important and the application of these properties can lead to behavior that is not observed in bulk devices. For example, solar cells made of nanocrystals can absorb nearly all of the incident solar radiation for wavelengths above their absorption onset with a film of only $200 \mathrm{~nm}$ thickness as a consequence of the increase in oscillator strength brought about by quantum confinement. In contrast, devices made of bulk inorganic semiconductors are generally several microns in film thickness.

The transport properties in thin film device structures made of conjugated polymers have been well characterized using space charge limited current models with field dependent mobilities $^{8}$. For the regioregular form of P3HT that is used in this study mobilities between $10^{-4}$ and $0.1 \mathrm{~cm}^{2} \mathrm{~V}^{-1} \mathrm{~s}^{-1}$ have been measured ${ }^{9-11}$, depending on the regioregularity of the polymer and the degree of order within the film. They cannot be fully described within the framework of the band model valid for bulk semiconductors ${ }^{12}, 13$. Nanocrystals are discrete particles, which can be physically separated from one another either by the surrounding medium or by a ligand shell. In fact, the temperature dependence of the conductivity in films of nanocrystals has been observed to be thermally activated, which suggests that an activated hopping transport model can be used to describe the transport of carriers ${ }^{14}$. This is similar to the hopping model described for organic 
conductors, but in this case, energetic disorder arises from the size distribution of the particles and geometric disorder from the separation of particles, spatially or by ligands. Unlike most conjugated polymer, nanocrystals can transport both electrons and holes with comparable mobilities $^{15}$.

Although, the individual transport properties of both nanocrystals and polymers have been studied separately in various electronic devices ${ }^{11,14-17}$, the carrier transport behaviour of these composite materials in composite devices, in particular photovoltaic cells, has not been sufficiently characterized. It is of particular interest to study transport in films of nanorodpolymer composites, as these systems represent a combination of the disordered transport in organic materials and the band like transport in inorganic semiconductors. Unlike the hopping charge transport that occurs between discrete nanocrystals, band conduction occurs within a single nanorod for an electron that is in the conduction band. The main effect of radial confinement in 1-D transport systems is enhanced scattering at the particle boundary, which reduces the carrier mobility. In this work, we investigate the transport of electrons and holes in CdSe nanorods and P3HT blend photovoltaics and provide a quantitative explanation for the observed electrical characteristics of these devices.

\section{EXPERIMENTAL METHOD}

Nanocrystals were synthesized using pyrolysis of organometallic precursors in a mixture consisting mainly of trioctylphosphine oxide (TOPO) and tributyl- or trioctylphosphine and small amounts of various phosphonic acids ${ }^{1,2,18}$. The recovered product was dispersed and washed three times in methanol to remove excess surfactant. Pyridine treatment of the nanocrystals to remove the surfactant used in the nanorod synthesis was accomplished by dissolving the particles in pyridine and subsequent precipitation in hexanes. Whereas TOPO 
coated CdSe nanocrystals are soluble in hexanes, pyridine-coated particles are insoluble in hexanes. Repeating the pyridine treatment two to three times can effectively replace more than $95 \%$ of the TOPO on the nanocrystal surface with pyridine ${ }^{19}$. The nanocrystals were characterized with transmission electron microscopy (TEM) using an FEI Tecnai 12 120kV microscope. Thin films of CdSe-P3HT blends approximately 50-100nm in thickness were investigated using TEM by casting a film on a $\mathrm{NaCl}$ IR window, floating the film in water and picking it up with a copper TEM grid. Film thicknesses were determined via atomic force microscopy (AFM) in tapping mode using a Nanoscope IIIa from Digital Instruments.

PV devices were fabricated by spin casting a solution of CdSe nanocrystals and P3HT in a pyridine-chloroform solvent mixture onto an indium tin oxide (ITO) or poly(ethylene dioxythiophene) doped with polystyrene sulfonic acid (designated PEDOT) on ITO coated glass substrate in an inert atmosphere, pumping for 12 hours under $<10^{-6} \mathrm{mbar}$ and evaporating aluminum on top to obtain the structure depicted in Fig. 1. Photocurrent measurements were completed using a 250W tungsten light source coupled to an Acton SP150 monochromator as an illumination source and a Keithley 236 Source Measure Unit to obtain current and voltage. The light intensity was measured with a calibrated Graseby silicon photodiode. The temperature dependence of the current-voltage characteristics was measured in vacuo at a pressure of about $10^{-5}$ mbar. Temperature was measured with a thermocouple that was in direct contact with the sample. For the intensity dependence of the PV response, an argon ion laser set at a wavelength of $514 \mathrm{~nm}$ was used. 


\section{THEORY}

\section{A. Equivalent Circuit Diagram}

Within a simple approach, a nanocrystal-polymer blend PV cell can be modelled as a diode in series with a resistor (the series resistor), and in parallel with a constant photocurrent source, a capacitor and another resistor (the shunt resistor) (see Fig. 1). For steady state measurements, the capacitance can be neglected.

Fig. 1

The shape of the I-V curve is strongly influenced by the value of the series resistance and the shunt resistance. Assuming that at high applied bias, the series resistance dominates and that there are minimal effects from space charge, which would decrease the applied field, the series resistance can be determined using Eq. 1. We can also calculate the shunt resistance using Eq. 2 in the case that the shunt resistance is substantially higher than the series resistance, which is the case for the devices discussed below.

$$
\begin{aligned}
& R_{s}=\lim _{V_{\text {out }} \rightarrow \infty}\left\{\frac{d V_{\text {out }}}{d I_{\text {out }}}\right\} \\
& R_{\text {sh }} \approx \frac{d V_{\text {out }}}{d I_{\text {out }}}\left(V_{\text {out }}=0\right) \quad R_{s}<<R_{\text {sh }}
\end{aligned}
$$

A low series resistance means that high currents will flow through the cell at low applied voltages and a high shunt resistance results if there are no shorts or leakages of photocurrent in the device. Contributions to the series resistance include contact resistances between the electrodes and the semiconductors as well as the bulk resistance of the blend in the PV device. To obtain a high fill factor for efficient power conversion, a series resistance approaching zero and a shunt resistance approaching infinity is desirable. 


\section{B. Simple Shockley Model}

Depending on the nature of the contacts, a number of mechanisms can affect the currentvoltage characteristics of a PV cell. Contacts between a metal and a semiconductor can either be ohmic or non-ohmic. The most important non-ohmic contact is a Schottky barrier. For a semiconducting device with one ohmic and one Schottky contact we can describe the currentvoltage characteristics by the Shockley equation ${ }^{20,21}$,

$$
I(V)=I_{0}\left(e^{\left(\frac{q V}{n k T}\right)}-1\right)
$$

where $I_{o}$ is the saturation current, $\mathrm{q}$ the magnitude of the electronic charge, $V$ the applied voltage, $n$ the ideality factor, $k$ Boltzmann's constant and $T$ the absolute temperature. The ideality factor $n$ takes account of recombination and tunneling processes and lies between 1 (ideal diode) and 2 (mainly recombination processes) for contacts between a metal and an inorganic semiconductor.

If we now replace the voltage $V$ in the Shockley equation by $V_{\text {out }}-I_{\text {out }} R_{s}$ and include series and shunt resistance, we obtain

$$
I_{\text {out }}=\frac{R_{\text {sh }}}{R_{s}+R_{\text {sh }}}\left[I_{0}\left(e^{\left(\frac{q\left(V_{\text {out }}-I_{\text {out }} R_{s}\right)}{n k T}\right)}-1\right)+\frac{V_{\text {out }}}{R_{\text {sh }}}\right]
$$

\section{Improved Shockley Model using Space Charge Approach}

At higher electric fields, there is a high current level resulting from injection of charges. If the charge carrier mobilities are low, space charge effects may become important. This space charge creates a field that opposes the applied bias and thus decreases the voltage drop across the Schottky diode. Using a modified Shockley equation similar to that used by Tagmouti et al. ${ }^{22}$ which incorporates the decrease in electric field due to space charge, we obtain the following equation: 


$$
I=\frac{R_{s h}}{1 / A+R_{s h}}\left[I_{0}\left(e^{\left(\frac{q\left(V-V_{b}\right)}{n k T}\right)}-1\right)+\frac{V}{R_{s h}}\right]
$$

The term $V_{b}$ accounts for both series resistance and space charge as follows,

$$
I=A V_{b}+B V_{b}^{2} \quad V_{b}=\frac{-A+\sqrt{A^{2}+4 I B}}{2 B} \quad \text { with } \quad A=1 / R_{s} \quad B=\frac{9}{8 d^{3}} a \mu \varepsilon \Theta
$$

In the above expression, $B$ accounts for the trap density $\Theta$, and the carrier mobility $\mu$ in a thin film with contact area $a$, thickness $d$, and permittivity $\varepsilon$. This model can also be used to describe nanocrystal-polymer devices for nanocrystals of various diameters and aspect ratios.

\section{Space Charge Limited Model}

In the case that the mobility of the active layer of the PV cell is very low, space charge effects are dominant throughout. Furthermore, for organic semiconductors the field dependence of the charge carrier mobility has to be taken into account as well ${ }^{8}$. The following equation for the space charge limited current with field dependent hole mobility is obtained ${ }^{8}$

$$
I=B e^{C \sqrt{V / d}} V^{2}
$$

where $\mathrm{B}$ and $\mathrm{C}$ are temperature dependent parameters (B as defined above) and $d$ is the thickness of the device.

\section{E. Numerical Methods}

To describe the experimental data presented in section IV using the three models outlined above least square fits were obtained numerically. Both Shockley models have three distinctive voltage regimes. At low applied bias the current was dominated by the shunt resistance $\mathrm{R}_{\mathrm{sh}}$; at intermediate electric field the basic Shockley equation given above in Eq. 3 is applicable, fixing the values of the saturation current $\mathrm{b}$ and the ideality factor $\mathrm{n}$; at high electric fields either series 
resistance for the simple model or the combined effect of the series resistance $R_{s}$ and space charge limited currents for the improved model dominate. As a result the number of free parameters in each regime is less than or equal to 2 in spite of an overall number of 4 fitting parameters (5 for the improved model).

\section{F. Modeling the Intensity Dependence of the Photocurrent}

To obtain information about the current limiting mechanism under illumination, it is useful to investigate the photocurrent as a function of incident light intensity. A simple model that describes the intensity dependence of the photocurrent in terms of the various modes of carrier recombination can be derived ${ }^{23}$. The density of photogenerated excitons $n$ is proportional to both the incident light intensity $I$ and the absorption of light in the material, so we have

$$
n=g \cdot I
$$

where $g$ is a constant proportional to the absorption of light in the material. Exciton decay is proportional to the density of bound electron-hole pairs (monomolecular recombination). Collection of both electrons and holes by the electrode is also a monomolecular process. In contrast, the recombination of an electron and a hole stemming from different electron-hole pairs will be proportional to the product of the electron and hole density. Charge recombination is therefore likely, if (i) the charge densities are large and (ii) if the density of electrons is on the same order of magnitude as the density of holes, which implies similar charge mobilities, given that electrons and holes are produced in equal numbers from a photo-excited exciton. If we assume that the electron mobility is equal to the hole mobility, the likelihood of non excitonic recombination is proportional to the square of the density of electron-hole pairs present, as every electron-hole pair can interact with all other electron-hole pairs present (this is called 
bimolecular recombination). Given that $1 / k$ and $1 / j$ are the monomolecular and bimolecular lifetimes, respectively, the change in electron-hole pair density is

$$
\frac{d n}{d t}=g \cdot I-k n-j n^{2}
$$

Using the steady state approximation, which is valid for current measurement under equilibrium conditions, the change in carrier density is zero. Thus,

$$
g \cdot I-k n-j n^{2}=0
$$

If $n$, which is proportional to the photocurrent, is small,

$$
n=\frac{g}{k} \cdot I
$$

Thus, for small light intensities a linear current-intensity relationship is predicted. For large $n$, i.e. for $k n \ll j n^{2}$, we obtain

$$
n=\sqrt{\frac{g I}{j}}
$$

In the case of large light intensities, we thus expect a square-root current-intensity relationship indicating the occurrence of bimolecular recombination.

\section{RESULTS AND DISCUSSION}

\section{A. Charge Transport in the Dark}

Fig. 2 shows TEM images of the two shapes of CdSe nanorods that have been used in devices. Fig. 3 shows the dark FV curve for a $90 \%$ by weight (wt.\%) $7 \mathrm{~nm}$ by $60 \mathrm{~nm}$ CdSe in P3HT device with PEDOT and aluminum as electrodes (designated Al:PEDOT). 
Fig. 2

\section{Simple Shockley Model}

We can fit the dark I-V characteristics of a nanorod-polymer device using the Shockley equation modified to take account of the series and shunt resistance for the low voltage range as shown in Fig. 3(a). Above 0.7V the fit deviates strongly from the data Fig. 3(a).

Fig. 3

\section{Improved Model}

The fit of the I-V characteristics of the nanorod-polymer device using the simple Shockley model is poor at high applied bias where there is a high current level resulting from injection of charges. Using a modified Shockley equation similar to that used by Tagmouti et al. $^{22}$ which incorporates the decrease in electric field due to space charge (Eq. 5), we can more effectively model the dark IV curve of the nanorod-polymer device as can be seen in Fig. 3(b). Thus, spacecharge effects are important for quantitatively describing the I-V characteristics at high bias indicating that low charge carrier mobilities limit transport of charges out of the device. The onset of space charge effects, arising from low carrier mobility within the film, creates a field that opposes the applied bias and thus decreases the voltage drop across the Schottky diode. Note that the Shockley equation does not only apply in the case of a simple Schottky diode, but also if there is recombination via traps (e.g. on the surface of the nanocrystals) in the depletion region (Shockley-Hall-Read recombination), or if the electron and hole charge densities are of the same order of magnitude ${ }^{21}$.

From the model, we obtain for the ideality factor, $n$, a value of 1.65 . While an ideality factor of 1 indicates that there are no current loss mechanisms, an ideality factor of 2 corresponds to current dominated by losses such as direct recombination or recombination via traps or mid gap 
states (bimolecular recombination) ${ }^{21}$. In real devices, loss mechanisms are important to consider, and a value of $n=1.65$ for our device is similar to values for $n$ found for photovoltaic cells made of bulk inorganic semiconductors. For bimolecular recombination to occur, the electron and hole densities have to be of similar order of magnitude in analogy to the discussion of the photocurrent intensity relationship in section III.F, with the difference that in the dark the charges are not produced from photo-excited excitons. In the case of nanorod-polymer films bimolecular recombination is likely to be caused by the presence of both holes that are injected from the PEDOT electrode into $\mathrm{P} 3 \mathrm{HT}$ and electrons injected into the nanocrystals from the aluminum electrode. Within the interpenetrating network, the two opposite charge carriers are likely to meet at a polymer-nanocrystal interface, where they can recombine non radiatively. Furthermore, structurally trapped electrons resulting from incomplete nanorod pathways provide non radiative recombination sites.

It should be added that we found some batch-to-batch difference in the Shockley fits resulting in values for $\mathrm{n}$ ranging from 1.5 to 1.9 . For a single device however the quality of the fit was much more sensitive to the value of $\mathrm{n}$, with similar quality fits falling typically in the interval $\mathrm{n} \pm 0.02$.

\section{Space Charge Limited Device}

By using Au and ITO as electrodes instead of Al:PEDOT, we observe a dramatic change in the shape of the dark FV curves (Fig. 4(a)). The resulting I-V curve cannot be modeled with the Shockley equation. However by using the space charge limited current model with field dependent mobility described in Eq. 6, a reasonable fit of the experimental data is obtained (Fig. 4(a)). As both $\mathrm{Au}$ and ITO are close to the highest occupied molecular orbital (HOMO) energy level of P3HT and far below the conduction band level of the nanocrystals (see Fig. 10(b)), it can 
be assumed that in the Au:ITO device the transport is dominated by holes through the polymer (Fig. 4(b)). Hole transport through P3HT in the present device therefore seems to follow the same mechanism as observed and extensively studied for other conjugated polymers such as poly(phenylene vinylene) (PPV $)^{8}$.

Fig. 4

As the Au:ITO and the Al:PEDOT devices do not show the same behaviour and the only difference between them is the possibility of electron injection from $\mathrm{Al}$ into the nanorods for the latter electrode combination, the transport of electrons seems to be responsible for the current voltage behavior of the device in Fig. 3. Though, even if the efficiency of transport in nanorodpolymer PV devices is enhanced over all-organic devices, the electron mobility is still limited by space charge effects at higher applied voltage (see Fig. 3(b)). In the case where a nanorod serves

as a continuous pathway for electron transport, space charge effects are likely to be minimal. However, because the nanorods are randomly dispersed within the polymer and their length is smaller than the device thickness, the particles do not span the entire thickness of the film and electrons are required to hop between nanorods. Space charge effects may be important in the case that hopping transport occurs, as the electron mobility is relatively low compared to bulk transport in this case. To realize the full potential of high carrier mobility in nanorods, it is necessary to synthesize longer rods and align them perpendicular to the substrate plane, such that electrons travel directly to the electrode.

\section{Temperature Dependence of Dark Current}

To determine the dependence of the dark FV characteristics on temperature a PV device using a composite of 90 wt.\% CdSe nanorod in P3HT was investigated under high vacuum at temperatures between $298 \mathrm{k}$ and $453 \mathrm{k}$. The temperature dependence of the deality factor $\mathrm{n}$ and 
the saturation current $\mathrm{I}_{0}$ which were obtained from fits to the $\mathrm{FV}$ characteristics using Eq. 4 are shown in Fig. 5.

Fig. 5

We can see that $\mathrm{n}$ is temperature dependent decreasing linearly throughout the temperature range investigated. As the electron mobility is likely to be thermally activated ${ }^{24}$ (due to hopping transport between nanorods), the transit time of the charges should decrease with increasing temperature resulting in less charge recombination and thereby a decrease in the ideality factor. We also observe an increase in photocurrent with temperature, which further suggests that recombination decreases with temperature. We note that this is in contrast to the Langevin type recombination that is observed in many polymeric semiconductors, which results in an ideality factor that increases with temperature ${ }^{25}$.

The corresponding Arrhenius plot of $\mathrm{I}_{0}$ vs. temperature is thermally activated (see Fig. 5(b)) with two activation energies and a transition at around $385 \mathrm{k}(1.47 \mathrm{eV}$ and $0.35 \mathrm{eV})$. The larger of the two is close to the band gap of the CdSe nanorods used in this study $(\sim 1.7 \mathrm{eV})$, whereas $0.35 \mathrm{eV}$ may be explained by the presence of trap levels in CdSe. Further work is needed to assign these activation energies with certainty.

\section{B. Charge Transport under Illumination}

With the knowledge of the dark I-V characteristics, we can already make some statements about the I-V characteristics under illumination. Under photovoltaic operation, the photocurrent results from absorption of a photon and subsequent creation of a weakly bound electron-hole pair, or exciton which then has to be split up into the free carriers, which in the present case occurs at the nanocrystal-polymer interface, and both the electron and the hole have to be transported to the respective electrodes. Both charges have to be collected to maintain charge 
neutrality of the device and the lower mobility of the two carriers limits the photocurrent. From the results of the previous section we can infer that the transport of holes in P3HT is limited to a greater extent (judging from the predominance of space charge effects) than the electron transport through the nanorods. For if the hole mobility in P3HT were larger than the electron mobility in the nanorods, the bipolar Al:PEDOT device would not show Shockley type behaviour and instead would have a similar characteristic as the hole only Au:ITO device. We would therefore expect that the photocurrent of the PV device is space charge limited just like the carrier with the lower mobility (the hole). Furthermore, this leads us to conclude that these hybrid polymer nanorod PV devices are limited by the hole mobility in the polymer, which is not the case in other polymer based photovoltaic cells. In these cells charge transport is limited by the electron mobility, which is typically extremely low for conjugated polymers due to the presence of ubiquitous electron traps such as oxygen ${ }^{26}$.

\section{Field Dependence of Photocurrent}

To model the charge transport characteristics of a conventional inorganic solar cell, it is typically possible to use the simple Shockley model of Eq. 4 and subtract a constant photocurrent $\mathrm{I}_{\mathrm{L}}$ from the modified Shockley characteristic on the right hand $\operatorname{side}^{21}$. In other words, the photocurrent for these cells does not depend on the applied bias. One way of measuring the photocurrent that is calibrated for the incident light intensity is the external quantum efficiency (EQE), which gives the number of electrons collected in the external circuit per incident photon.

Fig. 6 shows the EQE as a function of applied bias for the Al:PEDOT nanorod-polymer blend PV device investigated in the previous section. As can be seen, the photocurrent is dependent on the applied bias, which is not the case in conventional inorganic solar cells. This can be explained in the following way: while in the dark the current voltage characteristics are 
dominated by electron transport through the nanorods, both electrons and holes have to be transported out of the device under illumination. This means that the photocurrent is limited by the carrier with the lower mobility, which is almost certainly the hole transport in the polymer, as argued above. We therefore expect a photocurrent that, like the hole transporting P3HT, is dominated by space charge limited currents. Furthermore, the mobility of holes in P3HT is field dependent and so is the electron mobility in the case where electrons hop between nanorods via thermally activated hopping ${ }^{24}$. Under reverse bias, the applied field is in the direction of the built-in field and thus, the EQE increases. However, under forward bias, the applied voltage opposes the built-in field. With increasing forward bias, the field for charge separation and carrier mobility is decreased resulting in increased recombination of charges and, consequently, the $\mathrm{EQE}$ is seen to drop. In order to realize the full advantage of high electron mobility inorganic materials, it will thus be necessary to further improve the hole mobilities in the polymer or replace the polymer with a solution-processible inorganic semiconductor as the hole transporting component.

Fig. 6

\section{Influence of Illumination Intensity on Charge Transport}

As the density of charges within the photoactive medium depends on the number of photons absorbed, charge transport under illumination may depend on illumination intensity. By investigating the dependence of the most important photovoltaic parameters as a function of light intensity, we obtain valuable information about the nature of charge transport as well as the mechanism of charge recombination under illumination.

(a) External quantum efficiency and power conversion efficiency. The EQE at 514nm illumination at various light intensities of the 90 wt. $\% 7 \mathrm{~nm}$ by $60 \mathrm{~nm}$ CdSe nanorods in P3HT 
device is displayed in Fig. 7. The high $\mathrm{EQE}$ of $54 \%$ at the relatively low light intensity of $0.1 \mathrm{~mW} / \mathrm{cm}^{2}$ is only maintained up to an intensity of $10 \mathrm{~mW} / \mathrm{cm}^{2}$, above which the EQE decreases substantially. Note that sun light intensity according to the A.M. 1.5G standard (with spectral standard, ASTM E892 Global) corresponds to an intensity of $96.4 \mathrm{~mW} / \mathrm{cm}^{2}$. From Fig. 7, the $\mathrm{EQE}$ at this intensity is found to be only $37 \%$.

A measure for the overall conversion of the light's incident power into electrical power is given by the power conversion efficiency $\eta$, which is calculated according to the following equation

$$
\eta(\lambda)=\frac{I_{s c}(\lambda) \cdot V_{o c}(\lambda) \cdot F F(\lambda)}{P_{\text {light }}(\lambda)}
$$

where $I_{s c}, V_{o c}$ and $P_{\text {light }}$ stand for the short circuit current (which equals the photocurrent $I_{L}$ ), the open circuit voltage and the incident light power respectively. $\lambda$ indicates that these quantities are wavelength dependent; the fill factor $(\mathrm{FF})$ is defined as

$$
F F(\lambda)=\frac{\max _{0<V<V_{o c}}\{I(V(\lambda)) \cdot V(\lambda)\}}{I_{s c}(\lambda) \cdot V_{o c}(\lambda)}
$$

The intensity dependence of photocurrent, open circuit voltage and fill factor will be discussed separately below.

The power conversion efficiency as a function of light intensity shows a dependence that is similar to that observed for the $\mathrm{EQE}$ (Fig. 7). At a monochromatic intensity of $3 \mathrm{~mW} / \mathrm{cm}^{2}$, the device displays its maximum power conversion efficiency of $7 \%$.

Fig. 7

(b) A simple model for the intensity dependence of the photocurrent. The intensity dependence of the photocurrent (i.e. the short circuit current) is shown in Fig. 8. A linear fit of the log-log plot 
up to $60 \mathrm{~mW} / \mathrm{cm}^{2}$ yields a slope of 0.96 , corresponding to Current $\propto$ Intensity ${ }^{0.96}$. Unlike conventional inorganic PV cells, which exhibit a linear dependence for the current with light intensity, hybrid nanorod-polymer devices show a sublinear-dependence, especially above $10 \mathrm{~mW} / \mathrm{cm}^{2}$ light intensity. Thus, we face both a mo linear intensity dependence as well as a non linear field dependence of the photocurrent (see Fig. 6) and therefore models which have been successfully applied to fully polymeric cells, such as that used in Ref. 27, are not applicable in the case of our hybrid cell.

Fig. 8

The simple model described in III.F for the photocurrent-intensity relationship can be applied to explain the deviation from the monomolecular recombination mechanism at high incident light intensity. According to this model the sublinear photocurrent intensity relationship suggests that bimolecular recombination of carriers plays a more important role at high light intensities. Non-radiative decay between an electron and hole produced by different photons at the nanocrystal-polymer and the nanocrystal-nanocrystal interface becomes more likely at high light intensity, because the low mobility of the carriers results in a high density of electrons and holes in the nanorod-polymer device as the light intensity increases. At illumination below $10 \mathrm{~mW} / \mathrm{cm}^{2}$, monomolecular processes dominate the photocurrent. With the simple model for recombination, one cannot distinguish between carrier collection by the electrodes in the production of a photocurrent, and loss mechanisms such as radiative and non-radiative decay of excitons. Nevertheless, since monomolecular loss mechanisms do not cause the EQE to change with intensity, the decrease in EQE at high intensity observed in Fig. 7 may be explained by bimolecular recombination, but as we will see in the following section, this is not the only reason for the decrease in photocurrent. However, from the non-ideal Shockley characteristics in the 
dark with $\mathrm{n}=1.65$ discussed above, we know that bimolecular recombination is important for explaining charge transport in these cells. By comparing the current levels in Fig. 3 and Fig. 8, we obtain similar electron and hole densities in the dark and at high light intensity, assuming similar transit times. Bimolecular recombination should therefore be important at high light intensity.

By making the electron back transfer rate very slow compared to the forward rate, bimolecular lifetimes can be increased. The formation of a strong bond between nanocrystal and polymer through the use of a polymer with side groups that can bind to the nanocrystal surface can increase the orbital overlap between nanocrystal and polymer for fast electron transfer ${ }^{28-32}$. This and other similar approaches aimed at increasing bimolecular lifetimes in nanorod-polymer composite PV cells are currently under investigation within our laboratory.

Another strategy for decreasing bimolecular recombination is to lower the charge carrier density within the device. This can be accomplished by increasing the mobility of the carriers. Increasing the length of the nanorods as well as alignment of the particles in the direction of transport are possible methods for enhancing the electron mobility. Moreover, the hole mobility has been shown to increase as the regioregular P3HT chains become more ordered ${ }^{11}$.

(c) Fill factor and shunt resistance. The intensity dependence of the two remaining factors that contribute to the overall power conversion efficiency are displayed in Fig. 9(a). The $V_{o c}$ increases as the power is raised. The FF, on the other hand, rises at low intensity, as the power is increased, but then decreases again at intensities above $10 \mathrm{~mW} / \mathrm{cm}^{2}$.

Fig. 9 
A high device shunt resistance and low series resistance is required for obtaining a high fill factor. The dramatic decrease in fill factor at high intensity is a detriment to the performance of nanorod-polymer PV devices under high intensity such as solar conditions.

Fig. 10

A quantitative analysis of the inverse shunt resistance, or conductance, under increasing illumination corrected for the shunt resistance in the dark reveals that the photoconductance is proportional to the incident light intensity (see Fig. 10(a)). This means that the active material of the device behaves as a photoconductor. The presence of polymer or nanocrystal pathways that connect the anode to the cathode is a source of current leakage or electrical shorts, depending on the conductivity of the pathway. Carriers, which are transported to the electrodes via these routes, do not contribute to the photovoltage. In the dark, both materials exhibit very low intrinsic charge densities and consequently, the conductivity is low. As the intensity of illumination rises, the increased number of free electrons and holes enhances the conductivity of these pathways. Thus increased photoconductivity of the active layer is responsible for the decreasing fill factor at high light intensity. The high fill factors (>0.6), at solar intensities, that have been observed in bilayer devices made of organic materials can be explained by the absence of continuous pathways between the two electrodes ${ }^{33}$ whereas, single layer polymer devices have mostly demonstrated fill factors below $0.3^{3,34}$. This is understandable within the framework of photoconductivity. In an interpenetrating polymer blend, it is difficult to control the number of pathways that electrically short the device in the light and lower the shunt resistance. The addition of one thin, but continuous layer at the hole-collecting electrode and another at the electron-collecting electrode, as displayed in Fig. 10(b), can prevent the polymer and nanocrystal from shorting the two electrodes under illumination. The energy levels for the blocking layers 
must be chosen to allow for the photocurrent to be collected. A further indication in favor of this explanation is our observation of photoconductive gain in polymer/nanocrystal devices. Work is currently under way to improve the fill factor with this device structure.

We note that to remain within the framework of the equivalent circuit diagram, we have to replace the ohmic shunt resistor with a light intensity dependent resistor. From Fig. 1 we can infer that if the shunt resistance in the light approaches the value of the series resistance, a decrease in the EQE would ensue. This is the case for the device investigated here. From the photoconductance data, the value of the leakage current can be estimated, assuming that the built-in field is given by the open circuit voltage. By correcting the photocurrent data accordingly, it becomes clear that the photoconductive leakage current only accounts for part of the reduction of the current at high intensity. Bimolecular recombination, which was discussed in the previous section, is likely to be the source of the remaining reduction.

By improving the efficiency of $7 \mathrm{~nm}$ by $60 \mathrm{~nm}$ CdSe nanorod-polymer blend devices at high intensity and achieving the same EQE and FF as at low intensity, 54\% and 0.62 respectively, a power conversion efficiency of $3.6 \%$ can be reached under A.M 1.5G solar conditions, which is about double the value that is achieved at the present time ${ }^{7}$. Moreover, replacement of CdSe with CdTe to absorb a higher amount of solar radiation would enable these hybrid devices to reach a solar power conversion efficiency beyond $5 \%$.

(d) Open circuit voltage. The open circuit voltage is the voltage at which the photogenerated current is equal to the dark current, which flows in the opposite direction, such that no net current is detected in the external circuit. As the incident light intensity increases, the rising photocurrent produces a higher $\mathrm{V}_{\mathrm{oc}}$. The maximum $\mathrm{V}_{\mathrm{oc}}$ is determined by the difference between workfunctions of the electrodes aluminum and PEDOT as well as the difference between the 
lowest unoccupied energy level in the CdSe nanocrystal and the highest occupied energy level in $\mathrm{P} \mathrm{HT}^{35}$. This difference is governed by the diameter as well as the shape of the nanocrystal ${ }^{36}$. Given that the difference in workfunctions between PEDOT and aluminum is about $0.7 \mathrm{~V}$, it is expected that this would also be the maximum $\mathrm{V}_{\text {oc }}$. However, open circuit voltages of up to $0.78 \mathrm{~V}$ are observed in Fig. 11 for $3 \mathrm{~nm}$ by $60 \mathrm{~nm}$ nanorods, suggesting that the Fermi level of aluminum is partially pinned to the surface states close to the lowest unoccupied energy levels of CdSe. In many bulk inorganic semiconductors and metal junctions, the Fermi level of the metal is pinned to the surface states of the semiconductor within the band $\operatorname{gap}^{21}$. As a consequence, the observed barrier height, formed when two materials with differing Fermi levels are joined, for a Schottky contact between the metal and semiconductor does not vary with the workfunction of the metal. For a HOMO level of $5.1 \mathrm{eV}$ in P3HT and a Fermi level for PEDOT at $5.1 \mathrm{eV}^{37}$, we can calculate the effective workfunction for aluminum forming an interface with nanorod-polymer blends from the maximum open circuit voltage obtained for each nanocrystal size in Fig. 11 (see Table I). The observed trend in $V_{o c}$ agrees with the decrease in the band gap with increasing nanocrystal diameter and increasing nanorod length. The data for spherical nanocrystals (not shown) also falls within this trend with an upper limit to the open circuit voltage at $0.85 \mathrm{~V}$. Pinning of the metal cathode to the lowest unoccupied molecular orbital (LUMO) level of the polymer in organic optoelectronic devices is often observed and explained in terms of surface states and/or bonds between the organic and metal formed at the electrode interface ${ }^{38-42}$. The $V_{o c}$ in some polymer-based PV cells has also been found to be independent of the workfunction of the metal and more closely linked to the difference between the HOMO level of the electron donor and the LUMO level of the electron acceptor ${ }^{35,43,44}$.

Fig. 11 
Increasing the $\mathrm{V}_{\mathrm{oc}}$ by increasing the nanocrystal band gap is at the expense of expanding the device absorption into the far red. By decreasing the diameter of nanorods from $7 \mathrm{~nm}$ to $3 \mathrm{~nm}$, an $11 \%$ increase in $\mathrm{V}_{\mathrm{oc}}$ is obtained. However, $7 \mathrm{~nm}$ nanorods absorb $25 \%$ more solar radiation than $3 \mathrm{~nm}$ nanorods. Thus, higher efficiency can be gained through optimizing the absorption rather than the $\mathrm{V}_{\mathrm{oc}}$ in $\mathrm{CdSe}$.

\begin{tabular}{cc}
\hline \hline Nanocrystal Dimension & Effective Al Fermi level \\
\hline \hline $3 \mathrm{~nm}$ by $60 \mathrm{~nm}$ & $4.32 \mathrm{eV}$ \\
$7 \mathrm{~nm}$ by $60 \mathrm{~nm}$ & $4.40 \mathrm{eV}$ \\
\hline \hline
\end{tabular}

\section{TABLE I}

Because the internal field within the device is equal to zero at an applied voltage equal to the open circuit voltage, the field dependence of the photocurrent is of no importance and we can use the Shockley equation to model the $V_{o c}$ under various illumination intensities by the addition of a constant photocurrent term $I_{L}$ to Eq. 3. Resolving for $\mathrm{V}$ at I equal to zero, i.e. at the open circuit voltage, we obtain:

$$
V_{o c}=\frac{n k T}{q} \ln \left(\frac{I_{L}}{I_{0}}+1\right)
$$

Thus, if $I_{L} / I_{O} \gg 1$, a logarithmic relationship between open circuit voltage and the photocurrent can be expected, which is what we find in Fig. 9(b). By evaluating the logarithmic fit of the data we obtain $n=1.57$. This value is relatively close to the value of $n=1.65$ obtained using the Shockley fits in the dark, displayed in Fig. 3. This can be seen as an independent verification of the value of the ideality factor and in more general terms of the validity of the Shockley model. 


\section{Thickness Dependence of the Photoresponse}

As the film thickness increases from $100 \mathrm{~nm}$ to $350 \mathrm{~nm}$, the corresponding increase and subsequent decrease in EQE shown in Fig. 12(a) does not arise solely from an increase in absorbed light. The shape of the spectra depends on the thickness of the device and the photoresponse in the red regions of the spectra increases with thicker films. This can be attributed to a weak filter effect that results from part of the film not contributing to the photocurrent and acting as an optical filter ${ }^{35}$. In thick films, networks of physically touching nanorods transport electrons with low carrier mobility, compared to transport contained entirely in one particle, and electrons generated near the PEDOT electrode must traverse many nanorods to reach the collecting aluminum electrode. As a result blue light, which is absorbed closer to the transparent electrode, does not strongly contribute to the photocurrent ${ }^{35,} 45$. In addition, the electric field across the device responsible for charge separation is decreased at a given built-in voltage bias for a thicker film as compared to a thinner one.

Fig. 12

The competing effects of greater photon absorption and lower electric fields resulting in reduced charge separation efficiency and lower charge transport efficiency in a thicker film produces a maximum efficiency at $212 \mathrm{~nm}$ film thickness and optical density of 0.57 at $515 \mathrm{~nm}$ illumination (Fig. 12(a)), decreasing sharply at higher thicknesses. Polymer PV devices made by other research groups from blending a hole transport polymer with small molecules and other polymers have an optimized thickness of around $100 \mathrm{~nm}$, for at higher film thicknesses the filter effect becomes substantial. Blends of two polymers ${ }^{46}$, and $\mathrm{C}_{60}$ and polymer ${ }^{47}$ undergo gross phase separation above film thicknesses of $100 \mathrm{~nm}$ to produce inactive layers filter layers. In nanorod-polymer devices, we can make devices significantly thicker at $200 \mathrm{~nm}$ to achieve more 
absorption of light because the dispersion characteristics of the nanorods are well controlled and the transport properties of the nanorods are more efficient than for the above mentioned organic materials ${ }^{48}$.

We can also use the thickness dependence of the photovoltaic performance to study the sources of series resistance. The dependence of the series resistance on film thickness is not linear (Fig. 12(b)), suggesting that the bulk resistance of the film is not the only determining factor. The significant increase in series resistivity above a thickness of 200nm, correlates with the decrease in the overall EQE at higher film thickness seen in Fig. 12(b). The series resistance therefore seems to play an important part in determining the photocurrent. Moreover, there is a significant contribution from the conductivity of the electrode, both in terms of a contact resistance and a bulk resistance of the metals. The series resistance can be decreased significantly by decreasing the resistivity of the PEDOT electrode. By decreasing the bulk resistance of the electrode by three orders of magnitude from $10 \mathrm{k} \Omega / \mathrm{cm}$ to $5 \Omega / \mathrm{cm}$, the series resistivity of the device with thickness of $212 \mathrm{~nm}$ could be decreased from 93 to $50 \mathrm{k} \Omega / \mathrm{cm}^{2}$. Thus both the interface to the electrode as well as the bulk resistivity of the active layer play a role in determining the overall series resistance.

\section{CONCLUSIONS}

In conclusion, we have shown that PV cells made of nanocomposites of inorganic nanorods and a conjugated polymer can be quantitatively described using a Shockley type approach modified for taking account of shunt and series resistance, as well as space charge effects. This

organic-inorganic hybrid system shows both charge transport properties characteristic for low mobility disordered materials and bulk inorganic semiconductors. The applicability of the Shockley equation at low applied bias is characteristic for inorganic semiconductor devices, 
while the presence of space charge limited currents indicates that the low mobility of the carriers, especially holes, within the device limits transport and thus the performance of the PV device. In addition, under illumination the efficiency of photocurrent generation is found to be dependent on the applied bias unlike conventional inorganic semiconductor devices for which the photocurrent is a constant. Furthermore, the photocurrent-light intensity dependence was found to be sublinear which was explained in terms of a combination of bimolecular recombination and increased photoconductivity at high charge density. An analysis of the shunt resistance as a function of light intensity suggests that the photocurrent as well as the fill factor is diminished as a result of increased photoconductivity at the active layer at high light intensity. We suggest that the photocurrent-intensity relationship can be linearized by bringing the nanocrystals in closer contact to the polymer in order to make charge transfer faster in the forward direction, and by introducing blocking layers on either side of the device to reduce the effects of photoconductivity. By studying the intensity dependence of the open circuit voltage for nanocrystals with different band gaps, it was inferred that Fermi-level pinning occurs at the interface between the aluminum electrode and the nanocrystal. Moreover, the thickness dependence of the photoresponse is used to show that the series resistance of the device has contributions both from the bulk and the interface between semiconductor and metal electrode. In conclusion, this study shows that a theoretical understanding of the device physics of nanocrystal-polymer PV cells is essential for improving this class of materials for use in high light intensity applications such as solar cells.

\section{ACKNOWLEDGMENTS}

Supported by the National Renewable Energy Laboratory, grant XAD-9-18668-02 and the Department of Energy contract DE--AC03-76SF00098 and DE-AC03-76SF00098. We are 
grateful to the Robert D. Ogg Electron Microscopy Laboratory at the University of California, Berkeley for assistance with the TEM work. The authors would like to thank J. C. Scott for valuable discussions and W. C. Libby and G. L. Whiting for experimental assistance. WUH thanks the Natural Sciences and Engineering Research Council of Canada for a fellowship.

1 X. G. Peng, L. Manna, W. D. Yang, et al., Nature 404, 59 (2000).

2 Z. A. Peng and X. G. Peng, Journal of the American Chemical Society 123, 1389 (2001).

3 G. Yu and A. J. Heeger, Journal of Applied Physics 78, 4510 (1995).

4 J. J. Dittmer, R. Lazzaroni, P. Leclere, et al., Solar Energy Materials and Solar Cells 61, 53 (2000).

5 J. J. Dittmer, E. A. Marseglia, and R. H. Friend, Advanced Materials 12, 1270 (2000).

6 Y. Cui, X. F. Duan, J. T. Hu, et al., Journal of Physical Chemistry B 104, 5213 (2000).

7 W. U. Huynh, J. J. Dittmer, and A. P. Alivisatos, Science 295, 2425 (2002).

8 P. W. M. Blom, M. J. M. de Jong, and M. G. van Munster, Phys. Rev. B 55, R656 (1997).

9 A. Assadi, C. Svensson, M. Willander, et al., Applied Physics Letters 53, 195 (1988).

10 S. Marchant and P. J. S. Foot, Polymer 38, 1749 (1997).

11 H. Sirringhaus, P. J. Brown, R. H. Friend, et al., Nature 401, 685 (1999).

12 R. H. Friend, G. J. Denton, J. J. M. Halls, et al., Solid State Communications 102, 249 (1997).

13 R. H. Friend, R. W. Gymer, A. B. Holmes, et al., Nature 397, 121 (1999).

14 D. S. Ginger and N. C. Greenham, Journal of Applied Physics 87, 1361 (2000).

15 M. C. Schlamp, X. G. Peng, and A. P. Alivisatos, Journal of Applied Physics 82, 5837 (1997).

16 D. L. Klein, R. Roth, A. K. L. Lim, et al., Nature 389, 699 (1997). 
17 P. W. M. Blom and M. C. J. M. Vissenberg, Phys. Rev. Lett. 80, 3819 (1998).

18 X. G. Peng, J. Wickham, and A. P. Alivisatos, Journal of the American Chemical Society 120,5343 (1998).

19 M. Kuno, J. K. Lee, B. O. Dabbousi, et al., Journal of Chemical Physics 106, 9869 (1997).

20 J. Simon and J.-J. André, Molecular Semiconductors (Springer Verlag, Heidelberg, 1985).

21 S. M. Sze, The Physics of Semiconductor Devices (Johm Wiley \& Sons, New York, 1981).

22 S. Tagmouti, A. Outzourhit, A. Oueriagli, et al., Thin Solid Films 379, 272 (2000).

23 J. J. Dittmer, in Dye/Polymer Blends for Solar Cells (University of Cambridge, Cambridge, 2001), p. 148.

24 D. S. Ginger and N. C. Greenham, Synthetic Metals 124, 117 (2001).

25 P. W. M. Blom, M. J. M. deJong, and S. Breedijk, Applied Physics Letters 71, 930 (1997).

26 L. Bozano, S. A. Carter, J. C. Scott, et al., Applied Physics Letters 74, 1132 (1999).

27 D. Moses, J. Wang, G. Yu, et al., Physical Review Letters 80, 2685 (1998).

28 Y. Tachibana, J. E. Moser, M. Gratzel, et al., Journal of Physical Chemistry 100, 20056 (1996).

29 J. M. Rehm, G. L. McLendon, Y. Nagasawa, et al., Journal of Physical Chemistry 100, 9577 (1996).

30 H. N. Ghosh, J. B. Asbury, and T. Q. Lian, Journal of Physical Chemistry B 102, 6482 (1998).

31 Y. Q. Wang, J. B. Asbury, and T. Q. Lian, Journal of Physical Chemistry A 104, 4291 (2000).

32 J. B. Asbury, R. J. Ellingson, H. N. Ghosh, et al., Journal of Physical Chemistry B 103, 3110 (1999).

33 C. W. Tang, Applied Physics Letters 48, 183 (1986).

34 J. J. M. Halls, C. A. Walsh, N. C. Greenham, et al., Nature 376, 498 (1995).

35 R. N. Marks, J. J. M. Halls, D. D. C. Bradley, et al., Journal of Physics-Condensed Matter 6, 
1379 (1994).

36 L. S. Li, J. T. Hu, W. D. Yang, et al., Nano Letters 1, 349 (2001).

37 K. Z. Xing, M. Fahlman, X. W. Chen, et al., Synthetic Metals 89, 161 (1997).

38 W. R. Salaneck, Philosophical Transactions of the Royal Society of London Series aMathematical Physical and Engineering Sciences 355, 789 (1997).

39 M. Fahlman, W. R. Salaneck, S. C. Moratti, et al., Chemistry-a European Journal 3, 286 (1997).

40 E. Ettedgui, B. R. Hsieh, and Y. Gao, Polymers For Advanced Technologies 8, 408 (1997).

41 P. Dannetun, M. Boman, S. Stafström, et al., Journal of Chemical Physics 99, 664 (1993).

42 P. Dannetun, M. Lögdlund, C. Fredriksson, et al., Journal of Chemical Physics 100, 6765 (1994).

43 C. J. Brabec, A. Cravino, D. Meissner, et al., Advanced Functional Materials 11, 374 (2001).

44 C. J. Brabec, A. Cravino, D. Meissner, et al., Thin Solid Films 403, 368 (2002).

45 G. Yu, J. Gao, J. C. Hummelen, et al., Science 270, 1789 (1995).

46 J. J. M. Halls, in Cavendish Laboratory (University of Cambridge, 1997).

47 S. E. Shaheen, C. J. Brabec, N. S. Sariciftci, et al., Applied Physics Letters 78, 841 (2001).

48 W. U. Huynh, J. J. Dittmer, G. Whiting, et al., Advanced Functional Materials accepted for publication (2002).

\section{FIGURE CAPTIONS}

FIG. 1 The equivalent circuit diagram for a photovoltaic cell under illumination. Within a simple model a photovoltaic cell is a diode with a constant current source in parallel. $R_{s h}$ stands for the shunt resistance, $R_{S}$ is the series resistance, $C$ the capacitance and $V / I_{\text {out }}$ the current/voltage in the external circuit. 
FIG. 2 TEM image of (a) $3 \mathrm{~nm}$ by $60 \mathrm{~nm}$ and (b) $7 \mathrm{~nm}$ by $60 \mathrm{~nm}$ CdSe nanocrystals. Both images are at the same scale.

FIG. 3 The dark I-V curve for a 90 wt. $\% 7 \mathrm{~nm}$ by $60 \mathrm{~nm}$ CdSe in P3HT device with PEDOT and aluminum as electrodes is fitted with (a) the Shockley equation modified to take account of shunt and series resistance and (b) the space charge modified Shockley equation. Fits are solid lines. Also shown are the relative difference between the fit and the actual data.

FIG. 4 (a) The I-V characteristics of a 90 wt. $\% 8 \mathrm{~nm}$ by $13 \mathrm{~nm}$ CdSe nanocrystals in P3HT devices heated at $120^{\circ} \mathrm{C}$ with ITO as the front electrode and gold as the back electrode is fitted with space charge limited current equation. (b) Approximate energy levels of P3HT (dashed) and CdSe (solid line) and workfunctions for the electrodes used in this study.

FIG. 5 (a) The temperature dependence of the saturation current and the ideality factor n. A linear fit of the ideality factor is also shown. (b) The Arrhenius plot of the saturation current vs. temperature with the associated activation energies obtained from fits in the low and high temperature regimes.

FIG. 6 The EQE as a function of applied voltage for a 90 wt.\% 7nm by $60 \mathrm{~nm}$ CdSe in P3HT device with PEDOT and aluminum as electrodes. The EQE was calculated by subtracting the dark current from the light current. The illumination intensity was $\sim 0.1 \mathrm{~mW} / \mathrm{cm}^{2}$. Lines connecting the points serve as a guide to the eye. 
FIG. 7 Power conversion efficiency and EQE as function of 514nm light intensity of the 90 wt. \% 7nm by $60 \mathrm{~nm}$ CdSe nanocrystals in P3HT for the device for which dark current data is presented in FIG. 3.

FIG. 8 Current density as a function of $514 \mathrm{~nm}$ light intensity of the 90 wt. $\% 7 \mathrm{~nm}$ by $60 \mathrm{~nm}$ CdSe nanocrystals in P3HT device. A power law fit of this plot yields a slope of 0.96. The relative difference between the fit and data is shown in the inset.

FIG. 9 (a) FF and $V_{o c}$ as a function of $514 \mathrm{~nm}$ light intensity of the 90 wt. $\% 7 \mathrm{~nm}$ by $60 \mathrm{~nm}$ CdSe nanocrystals in P3HT device. (b) The $V_{o c}$ as a function of photocurrent density of the same device. A fit to the Shockley equation is shown.

FIG. 10 (a) Photoconductance given by $1 /\left(R_{\text {sh }}\right.$ light $\left.-R_{\text {sh dark }}\right)$ as a function of $514 \mathrm{~nm}$ light intensity of the 90 wt. \% $7 \mathrm{~nm}$ by $60 \mathrm{~nm}$ CdSe nanocrystals in P3HT. A linear fit of the data is shown. (b) Energy level diagram and device schematic for nanocrystal-polymer photovoltaic cell with electron and hole blocking layers.

FIG. 11 The $V_{o c}$ as a function of 514nm light intensity of 90 wt. \% CdSe nanocrystals in P3HT devices for nanocrystals of dimensions $3 \mathrm{~nm}$ by $60 \mathrm{~nm}$ (filled circles) and $7 \mathrm{~nm}$ by $60 \mathrm{~nm}$ (empty squares). 
FIG. 12 (a) The EQE spectra for a series of 90 wt. $\% 7 \mathrm{~nm}$ by $60 \mathrm{~nm}$ CdSe in P3HT devices with different film thicknesses. (b) Integral of the photocurrent spectra for the devices in FIG. 12 and series resistivity for $90 \mathrm{wt} . \% 7 \mathrm{~nm}$ by $60 \mathrm{~nm}$ CdSe in P3HT for the electrode pair Al:PEDOT at various film thicknesses. Lines connecting the points serve as a guide to the eye.

Table I Effective workfunction, calculated from the $V_{o c}$ for aluminum electrodes of 90 wt.\% nanocrystals in polymer blend devices for various nanocrystals. 


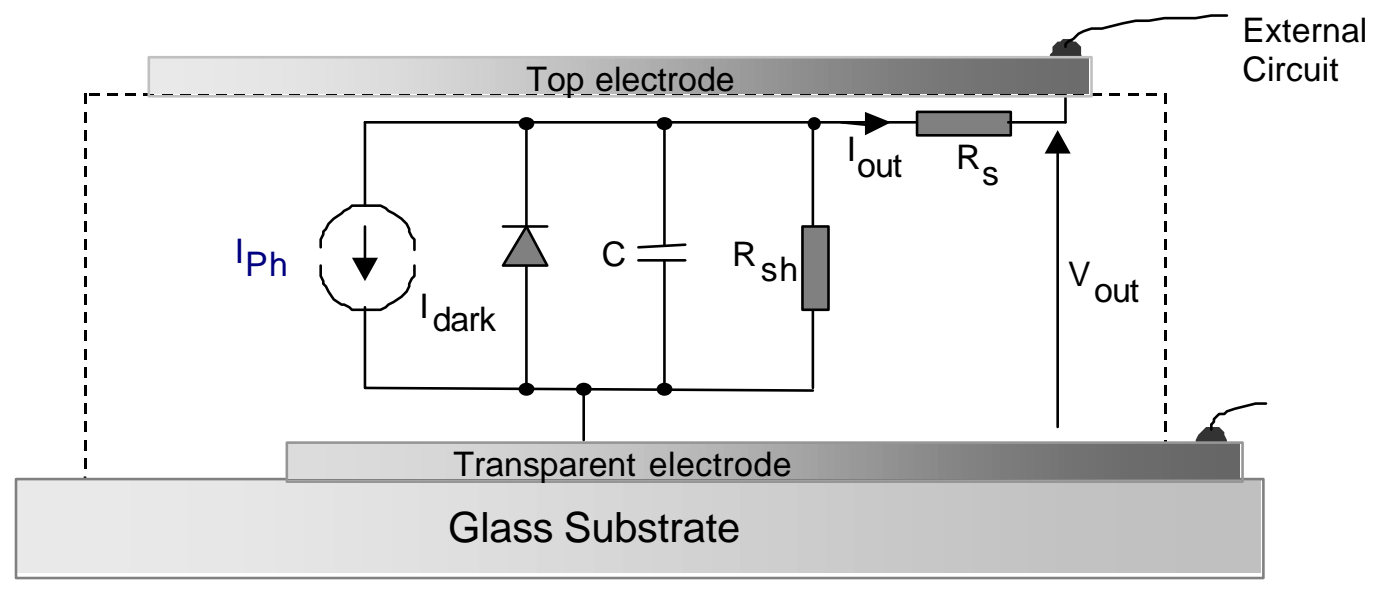

Fig. 1 


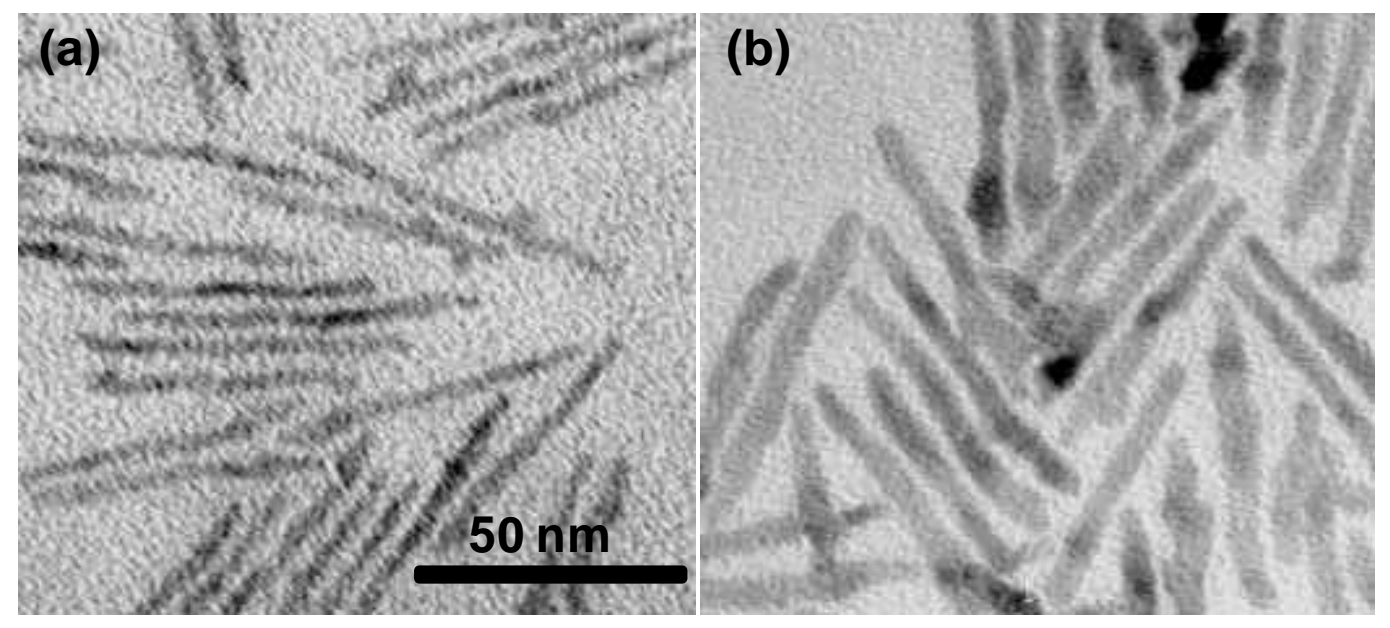

Fig. 2 

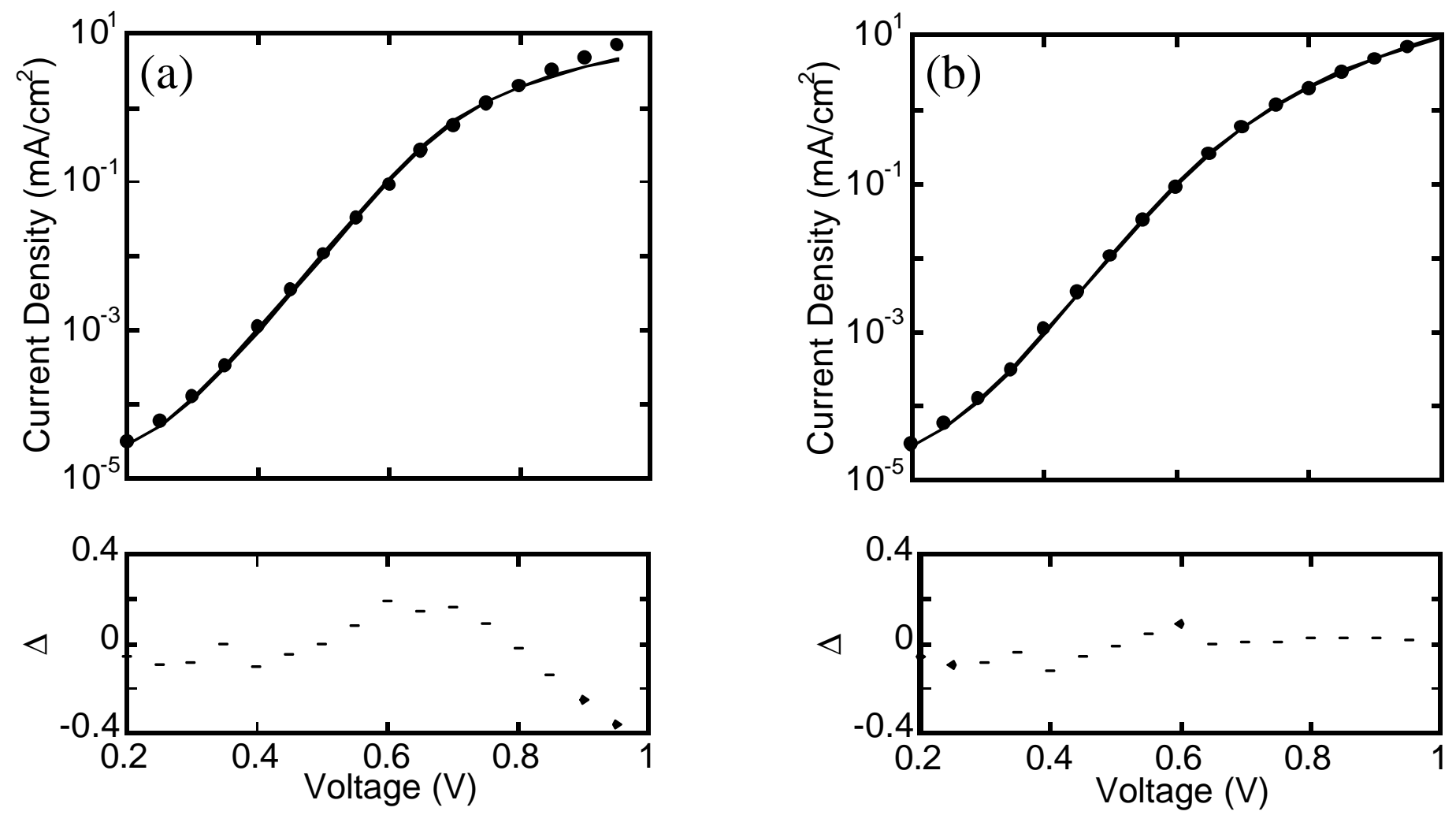

Fig. 3 


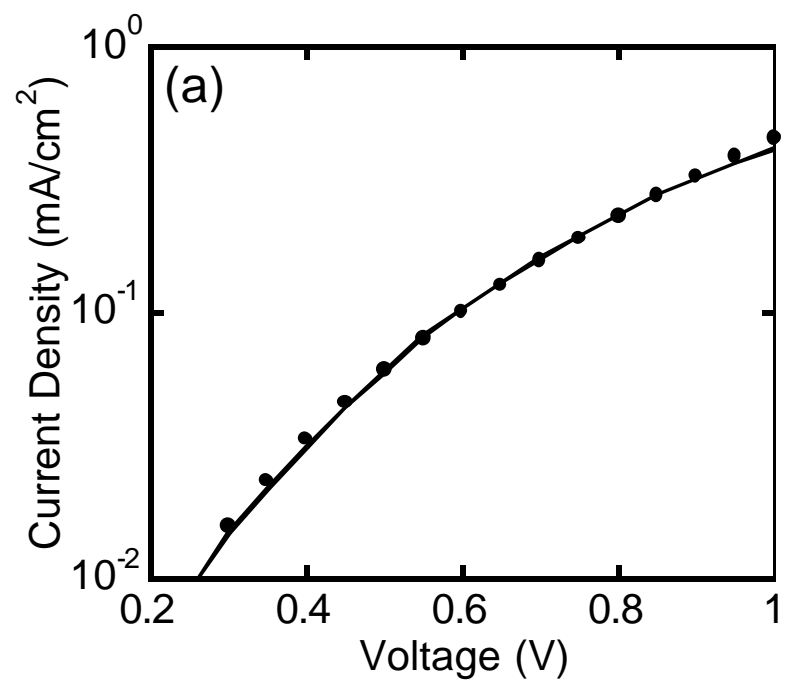

(b)



Fig. 4 

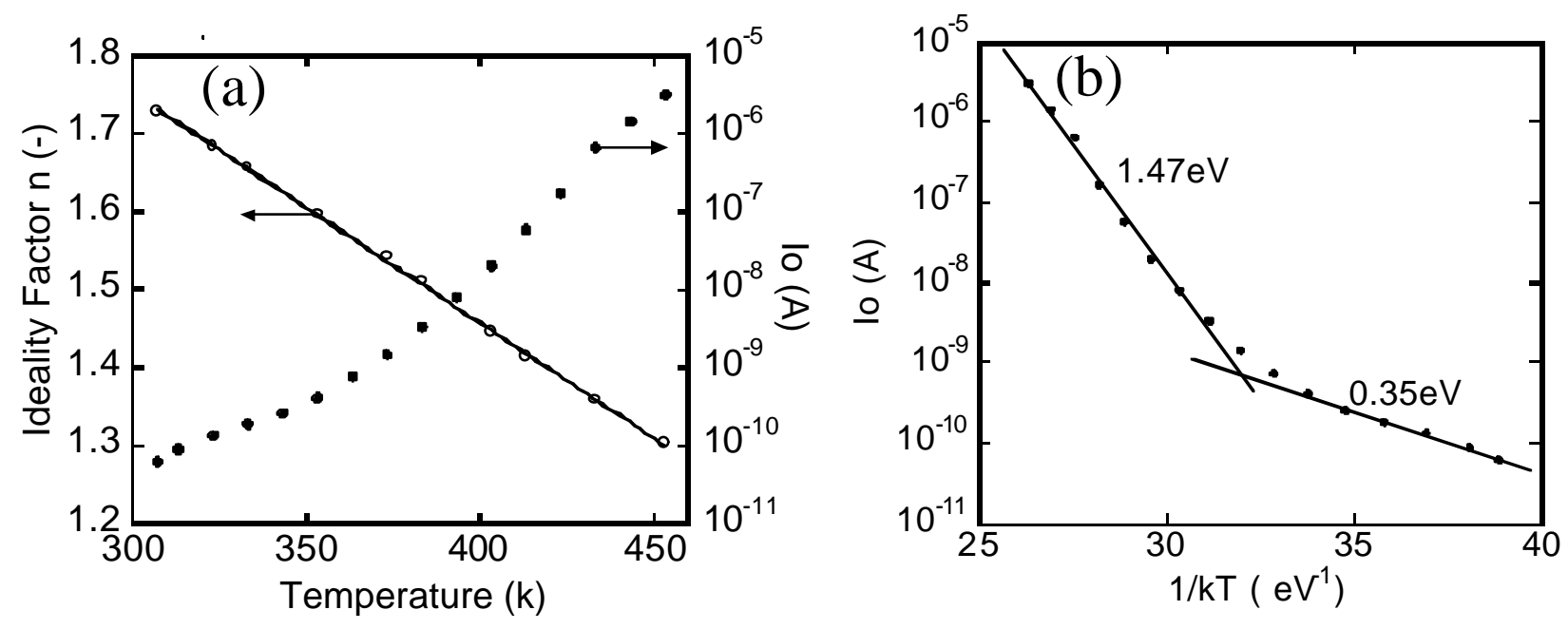

Fig. 5 


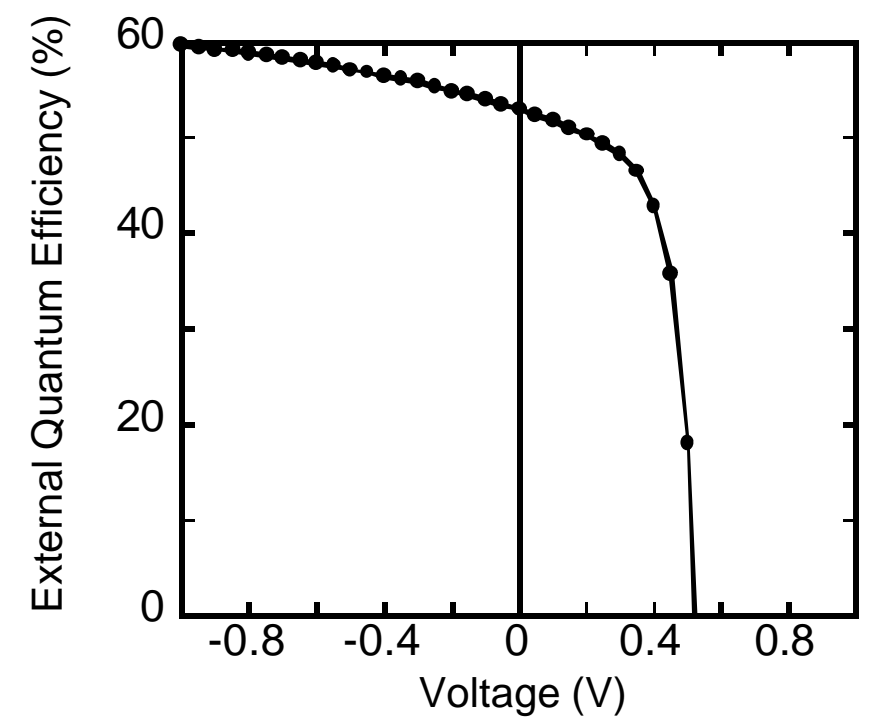

Fig. 6 




Fig. 7 




Fig. 8 

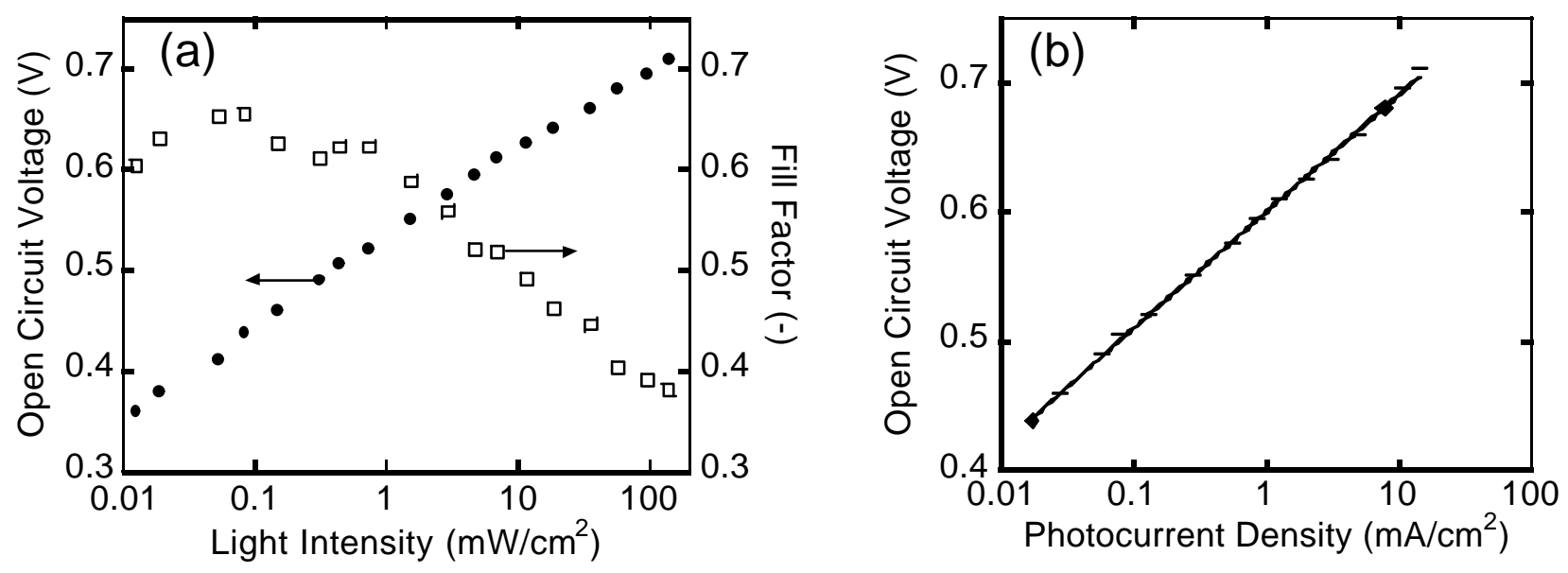

Fig. 9 


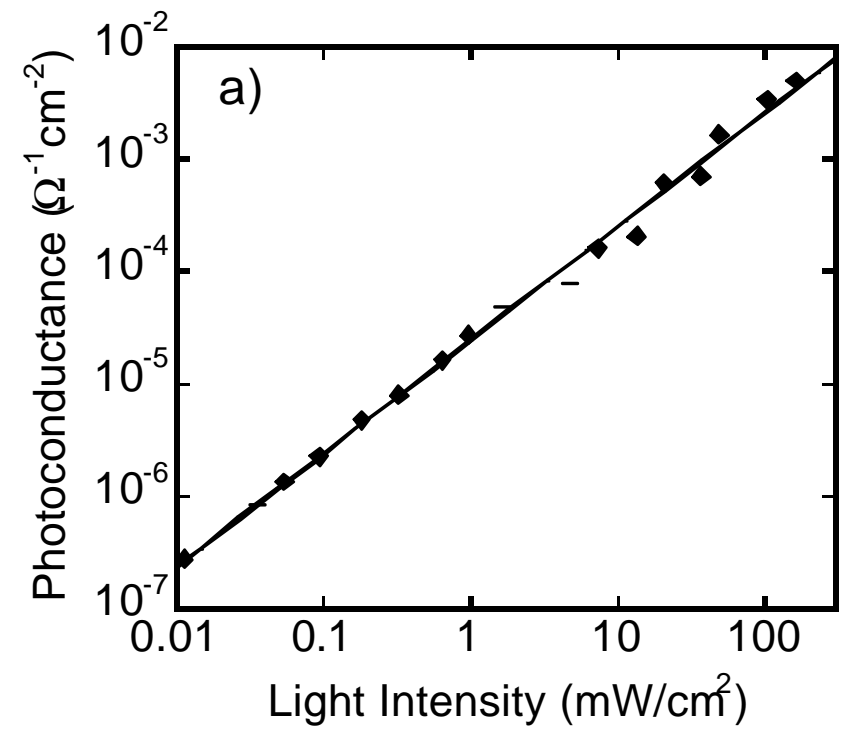

b)



Hole -blocking

Fig. 10 


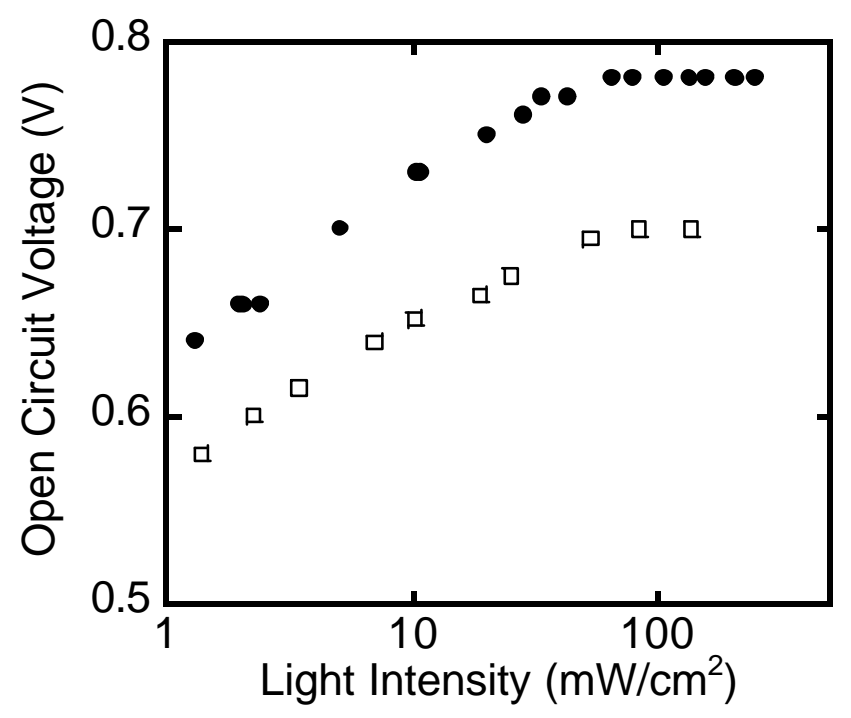

Fig. 11 

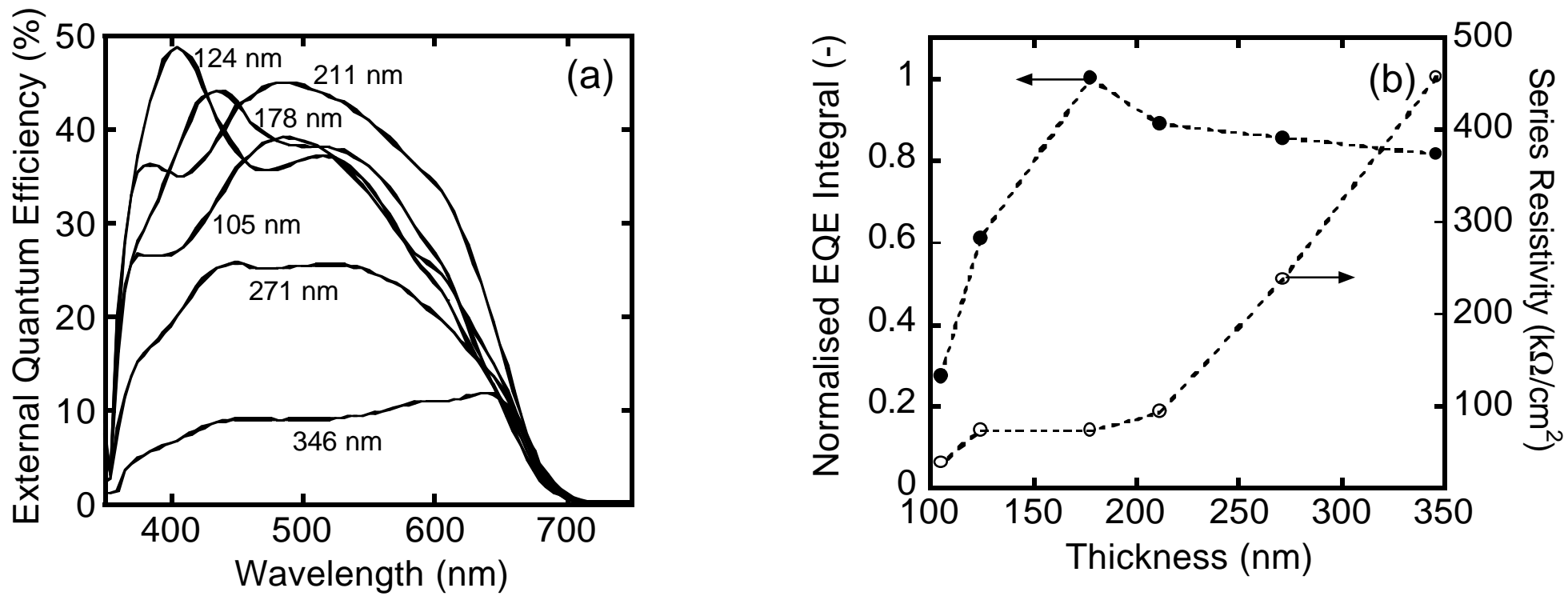

Fig. 12 